\title{
Fast dynamic imaging technique to identify obstructive lesions in the CSF space: report of 2 cases
}

\author{
Doris D. Wang, MD, PhD, ${ }^{1}$ Kenneth W. Martin, MD, ${ }^{2}$ Kurtis I. Auguste, MD, ${ }^{1,3}$ and Peter P. Sun, MD ${ }^{1,3}$ \\ ${ }^{1}$ Department of Neurological Surgery, University of California, San Francisco; and ${ }^{2}$ Department of Radiology and ${ }^{3} \mathrm{Children's}$ \\ Neurosurgical Associates, UCSF Benioff Children's Hospital, Oakland, California
}

\begin{abstract}
Disorders of CSF dynamics such as syringomyelia and obstructive hydrocephalus can be caused by thin mobile obstructive lesions not visible on traditional MRI sequences. New imaging techniques with balanced steady-state free precession (bSSFP) and dynamic imaging with bSSFP cine allow visualization of these pulsatile structures within the CSF space. The authors present 2 cases involving pediatric patients - one who developed presumed idiopathic syringomyelia and one with presumed communicating hydrocephalus in association with Pfeiffer syndrome - who harbored thin dynamic obstructive lesions seen on bSSFP cine studies using 1.5-T MRI.

In combination with traditional CSF cine studies and bSSFP, bSSFP cine sequence was able to detect dynamic membranous adhesions not seen on traditional MRI sequences. These previously undetectable lesions on traditional MRI sequences were the etiology of CSF obstruction, and tailored surgical approaches were performed to avoid shunting in both patients. These reports demonstrate the clinical utility for using these novel imaging tools for the detection of thin adhesions and dynamic lesions in the central nervous system. Balanced SSFP cine sequences can supplement conventional MR modalities to identify these otherwise poorly visualized lesions responsible for presumed communicating hydrocephalus or idiopathic syringomyelia.

http://thejns.org/doi/abs/10.3171/2014.11.PEDS13620
\end{abstract}

KEY WORDS steady-state free precession; gradient echo MRI; CSF flow; CSF cine; CSF obstruction; hydrocephalus; syrinx; syringomyelia; technique

$\mathrm{D}$ ISORDERS of CSF dynamics such as syringomyelia and hydrocephalus are relatively common disorders in children and frequently involve obstruction of CSF flow. CSF flow obstruction often can be caused by thin arachnoid adhesions or membranes. While effects of CSF obstruction can manifest as ventriculomegaly and syringomyelia that are easily identifiable on traditional T1and T2-weighted MRI, the thin lesions themselves, which can be pulsatile, are nearly impossible to visualize with these conventional imaging techniques.

Recently, new MRI sequences have been developed which may offer a solution. Steady-state free precession (SSFP) is a type of gradient-echo steady-state coherent MRI that produces high spatial resolution images with high signal-to-noise and high contrast-to-noise ratios. This sequence utilizes a very short repetition time, employs radiofrequency pulses with a large flip angle, and balances gradients in all 3 directions. ${ }^{19}$ Commercial names for bal- anced SSFP (bSSFP) include: fast imaging employing steady-state acquisition (FIESTA) sequence for GE scanners, balanced fast field echo (bFFE) for Philips, and true fast imaging with steady-state precession (TrueFISP) for Siemens. These gradient echo sequences yield high signal of blood vessels and body fluid without using contrast material and can be used to highlight signals from the CSF space. ${ }^{18}$ MR cisternography using bSSFP sequence can effectively visualize the neurovascular structures within the cerebellopontine cistern without CSF pulsation artifacts in a short examination time. ${ }^{9,14}$ Balanced SSFP has been demonstrated to be extremely useful for detailed anatomy of the cisternal segments of the lower cranial nerves as well as cerebellopontine angle lesions. ${ }^{13,19}$ Balanced SSFP is also useful in detecting neurovascular compression of the trigeminal nerve. ${ }^{12}$

However, bSSFP and other traditional MRI techniques are limited by their susceptibility to motion artifacts caused

ABBREVIATIONS bSSFP = balanced SSFP; ETV = endoscopic third ventriculostomy; SSFP = steady-state free precession.

SUBMITTED November 19, 2013. ACCEPTED November 7, 2014.

INCLUDE WHEN CITING Published online February 27, 2015; DOI: 10.3171/2014.11.PEDS13620.

DISCLOSURE The authors report no conflict of interest concerning the materials or methods used in this study or the findings specified in this paper. 
by heartbeat, breathing, or swallowing. While bSSFP is effective at detecting detailed structures within the CSF space, dynamic thin membranous adhesions are difficult to visualize using a non time-resolved approach since they move synchronously with CSF pulsations. ${ }^{716}$ A cardiac gated sequence called bSSFP cine has been reported to be an effective method for the functional diagnosis of cardiac disease. ${ }^{1,2}$ Similar to bSSFP, bSSFP cine uses shorter echo time relative to the repetition time. Balanced SSFP cine has been used in abdominal imaging as well as arteriography and venography to visualize structures that move with the cardiac cycle., $, 5,11,15,18$ A recent report showed that cardiac-gated bSSFP cine was able to detect pulsatile arachnoid spinal adhesions not seen on other MRI sequences in an adult patient with presumed idiopathic syringomyelia. ${ }^{6}$ This raises the possibility for its broader application in the pediatric population as part of the evaluation for dynamic CSF disorders.

Here, we present 2 cases-one involving a child with presumed idiopathic syringomyelia and the other involving a child with presumed communicating hydrocephalus - in which bSSFP and bSSFP cine studies were performed to search for dynamic obstructive lesions. Using these imaging techniques, we were able to localize the adhesions causing the CSF flow obstruction and tailor the surgical treatments to avoid shunting. These illustrative cases show that bSSFP may have great clinical value in the preoperative evaluation for conditions of altered CSF dynamics to localize obstructive lesions.

\section{Case Reports \\ Case 1}

This 10-year-old boy with idiopathic syringomyelia had been born with ventriculomegaly and had undergone ventriculoperitoneal shunt placement shortly after birth. At 2 years of age, he had a shunt infection requiring shunt externalization. After a course of antibiotic treatment, a new shunt was placed, but his postoperative course was complicated by overdrainage and development of subdural hygromas. The shunt was revised again, but at 4 years of age, the patient developed slit ventricles requiring multiple additional shunt revisions. Placement of a programmable valve resulted in a return to normal ventricle size and good shunt function. At 6 years of age, he developed back pain, bilateral lower-extremity fatigue, and hip pain with intermittent hand numbness. MRI of the spine showed a cervicothoracic syrinx extending from C-3 to T-6 without a Chiari malformation (Fig. 1A). Shunt evaluation revealed a functional and patent ventriculoperitoneal shunt. When he was 10 years old, his back and bilateral lower-extremity pain and weakness worsened and the syrinx progressed, and he therefore underwent cardiac gated phase-contrast CSF flow, bSSFP, and bSSFP cine studies. The CSF cine study revealed flow disturbance near the dorsal cervicomedullary junction with abnormally increased pulsatility of the medulla and brainstem (Fig. 1C and Video 1).

VIDEO 1. Case 1. Video clip from the CSF cine study demonstrating CSF flow obstruction at the dorsal cervical cord region. Copyright Doris D. Wang. Published with permission. Click here to view with Media Player. Click here to view with Quicktime.
A bSSFP study performed on a 1.5-T Phillips MR scanner revealed hypointense signal just caudal to the obex, but due to motion artifacts, a lesion could not be identified (Fig. 1E). Remarkably, when the bSSFP cine sequence was used, a mobile, thick, membrane-like structure extending from the cervical cord at the $\mathrm{C}-1$ level dorsally to the thecal sac could be easily distinguished (Fig. 1F and Video 2).

VIDEO 2. Case 1. Video clip from the cardiac-gated bSSFP cine sequence demonstrating mobile membrane at the dorsal cervical cord responsible for the syringomyelia. Copyright Doris D. Wang. Published with permission. Click here to view with Media Player. Click here to view with Quicktime.

In addition, there appeared to be a thinner membrane extending from the obex to the cerebellar tonsils. Together, these studies suggested that the syrinx was mostly likely due to obstruction of normal CSF flow dynamics at the craniocervical junction from this membrane. The patient underwent a suboccipital craniectomy and C-1 laminectomy. Intraoperatively, an arachnoid membrane was clearly visualized adhering to the dura at the dorsal cervical cord region (Fig. 1D). After lysis of the adhesions, the patient had complete resolution of his cervicothoracic syrinx and symptoms (Fig. 1B). He has been followed with serial imaging for the last 24 months with no clinical or imaging evidence of recurrent syringomyelia.

\section{Case 2}

This 10-year-old boy with Pfeiffer syndrome presented with hydrocephalus. He had been born with severe facial dysmorphism, and although a head CT performed shortly after his birth showed open sutures, by 16 months he had developed cranial pansynostosis. He underwent calvarial vault expansion and frontoorbital remodeling. Imaging performed in the perioperative period showed no hydrocephalus. However, at 6 years of age he developed headaches, and a CT scan revealed ventriculomegaly, which was presumed to be due to communicating hydrocephalus. Standard MRI sequences revealed a mild Chiari malformation and a stable posterior fossa arachnoid cyst (Fig. $2 \mathrm{~A}$ ), and a right occipital ventriculoperitoneal shunt was placed. The patient subsequently underwent Le Fort III midface osteotomy and midface advancement.

He was doing well until he presented with severe headache and fever and was found to have community-acquired Streptococcus pneumoniae meningitis from CSF cultures at the age of 10 years. A shunt tap confirmed the infection. The shunt was removed and an external ventricular drain was placed. He was treated with intravenous antibiotic therapy. The external ventricular drain continued to have high output (400-500 ml/day) despite weaning, and he underwent additional imaging for further evaluation. A CSF cine study showed flow obstruction from the sylvian aqueduct to the fourth ventricle with flow through the foramen magnum (Fig. 2B and Video 3).

VIDEO 3. Case 2. Video clip from the CSF cine study indicating CSF flow obstruction at the sylvian aqueduct. Copyright Doris D.

Wang. Published with permission. Click here to view with Media

Player. Click here to view with Quicktime.

T1-weighted MRI, however, did not reveal aqueductal stenosis (Fig. 2A). Balanced SSFP MRI showed narrowing 

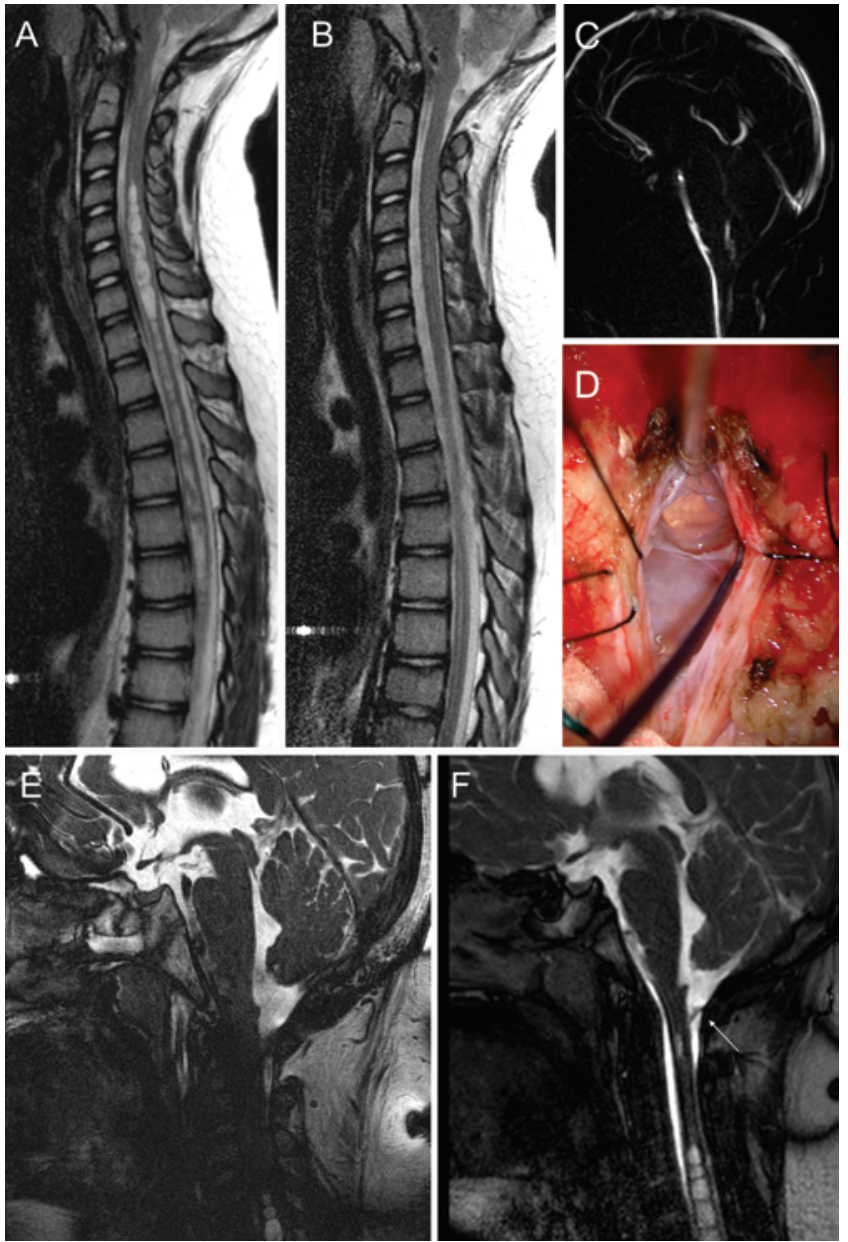

FIG. 1. Case 1. Idiopathic syringomyelia in a 10-year-old boy with shunttreated hydrocephalus. A and B: Preoperative (A) and postoperative (B) T2-weighted sagittal cervical and thoracic spine images showing resolution of cervicothoracic syringomyelia after surgery. Note that the preoperative MR image does not show any obvious lesion causing CSF obstruction. C: Sagittal image from the preoperative CSF cine flow study demonstrating obstruction of CSF flow at the cervicomedullary junction. D: Intraoperative photograph demonstrating the arachnoid adhesions at the obex. E and F: Sagittal bSSFP image (E) showing signal hypointensity in the dorsal cervical cord below the obex, and sagittal image from the dynamic bSSFP cine study (F) showing the thick membrane (arrow) causing the CSF flow obstruction that was visualized intraoperatively (seen in D). Figure is available in color online only.

of the aqueduct (Fig. 2C) but did not definitively identify the obstructive lesion for performance of an endoscopic third ventriculostomy (ETV). On the other hand, bSSFP cine imaging revealed a membrane between the anterior tectum and the dorsal midbrain occluding the sylvian aqueduct (Fig. 2D and Video 4).

VIDEO 4. Case 2. Video clip from the cardiac-gated bSSFP cine sequence demonstrating adhesion between dorsal midbrain and tectum. Copyright Doris D. Wang. Published with permission. Click here to view with Media Player. Click here to view with Quicktime.

Given the definitive imaging evidence of aqueductal obstruction, an ETV was performed. This procedure successfully treated the patient's hydrocephalus, and as of this writing, he has had no clinical or imaging evidence of recurrent hydrocephalus for 20 months.
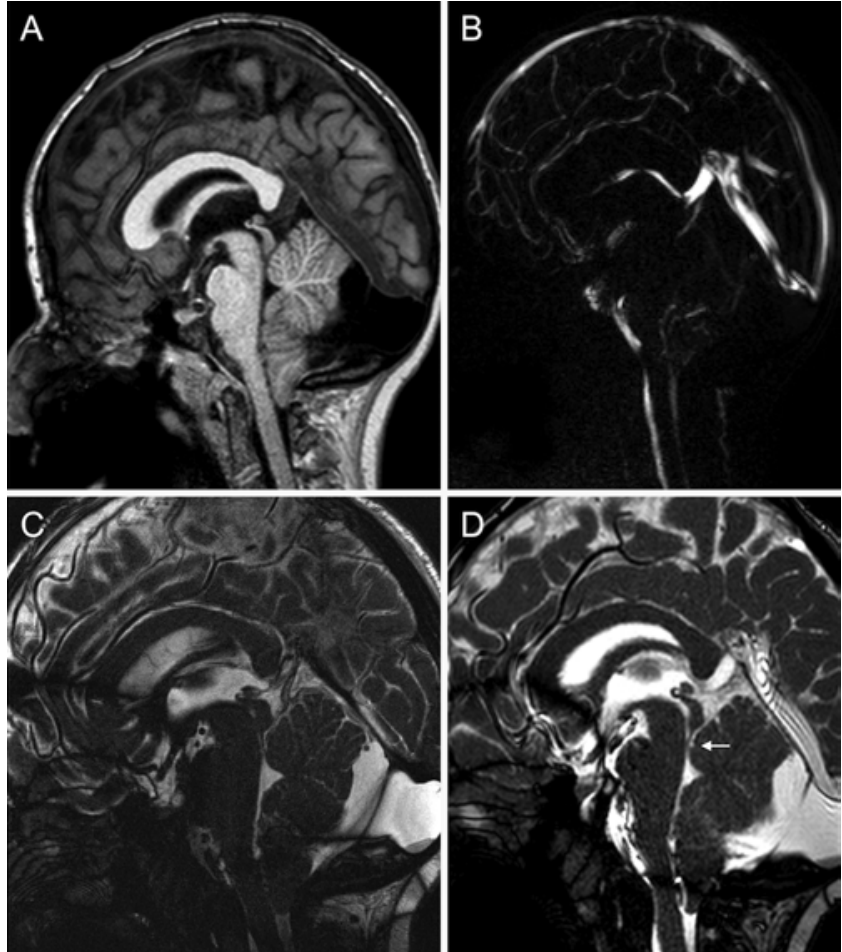

FIG. 2. Case 2. Obstructive hydrocephalus in a 10 -year-old boy with Pfeiffer syndrome. A: Sagittal T1-weighted MR image showing no obvious aqueductal stenosis. B: Sagittal image from the CSF cine study demonstrating CSF flow obstruction near the sylvian aqueduct. C: Sagittal bSSFP image showing narrowing of the cerebral aqueduct. D: Sagittal image from the dynamic bSSFP cine study better demonstrating the narrowing of the cerebral aqueduct. Note the adhesion between dorsal midbrain and tectum (arrow).

\section{Discussion}

Advances in neuroimaging techniques have been essential in the diagnosis and treatment of central nervous system disorders. With the advent of high-resolution, high-contrast MRI sequences, we are now able to discern small vessels, cranial nerves, and other structures that were once difficult to visualize. Here, we introduce the use of gradient echo sequences bSSFP and bSSFP cine study to evaluate dynamic lesions of the central nervous system that are extremely difficult to visualize on conventional MRI sequences.

These imaging techniques have potential widespread clinical value in identifying dynamic CSF obstructive lesions in pediatric patients in order to avoid the need for CSF shunting. As shown by these 2 illustrative cases, identifying dynamic obstructive lesions not seen on standard MRI sequences can alter the surgical management. In the case of syringomyelia, the pathophysiology usually involves CSF flow alteration or spinal cord tethering. However, obstructive lesions caused by arachnoid membranes cannot be directly identified by means of standard MRI techniques due to their mobile nature. ${ }^{10,17}$ Without removal or fenestration of the lesion causing flow obstruction, placement of syringopleural or syringosubarachnoid shunts is the standard treatment for syringomyelia. However, insertion of these shunts may be associated with morbidity, and the shunts are often prone to failure. Us- 
ing bSSFP and bSSFP cine makes it possible to identify mobile adhesions and visualize their pulsations in real time, allowing for direct surgical treatment of the CSF obstruction without shunting. In our Case 1, it is interesting to note that the obstructive lesion was rostral to the syrinx at the craniocervical junction, which has also been described in cases where syringomyelia has improved following craniocervical decompression in patients without hindbrain herniation (Chiari 0). ${ }^{8}$ In some Chiari 0 patients, a fourth ventricular veil and intradural adhesions were encountered intraoperatively. Surgical lysis of these lesions can lead to resolution of the syringomyelia. While these lesions themselves were not seen on standard MRI, they may now be visualized with bSSFP.

In the diagnosis of hydrocephalus, using bSSFP and bSSFP cine in combination may have clinical value in detecting lesions that cause CSF flow obstruction. The etiology of hydrocephalus in craniosynostosis is thought to be variable and multifactorial. ${ }^{3}$ The patient in our Case 2 was assumed to have communicating hydrocephalus based on a standard MRI study. Although there was a mild Chiari malformation, in the absence of aqueductal stenosis, the patient was initially treated with a ventriculoperitoneal shunt. However, further imaging with bSSFP suggested that there was an aqueductal obstruction, and bSSFP cine was able to confirm the presence of obstructive aqueductal lesion or stenosis not seen on the previous MRI. With the diagnosis of obstructive hydrocephalus, the patient was able to undergo successful treatment with an ETV instead of ventriculoperitoneal shunt placement.

The combination of CSF cine, bSSFP, and dynamic bSSFP cine studies offers several advantages. While cine study can detect CSF flow or obstruction, the image resolution is extremely poor. Balanced SSFP can accurately detect thin membranous adhesions not identifiable on traditional T1- and T2-weighted MR images. However, in the case of dynamic lesions, mobile membranes will appear as streak artifacts on bSSFP sequences. Cardiacgated bSSFP cine sequences, on the other hand, have lower resolution compared with bSSFP, but their ability to discern dynamic components is uniquely useful. In addition, bSSFP sequences are relatively quick to obtain on a 1.5-T MR scanner; a cardiac-gated bSSFP sequence has an acquisition time of 1.5 minutes and does not require intravenous contrast administration. Therefore, when used in combination, these imaging techniques can not only detect CSF flow obstructions, but also localize the lesion, even down to the thinnest arachnoid adhesions. Application of bSSFP sequences can identify obstructive lesions and allow for effective direct surgical treatment of these lesions without the need for CSF shunting. Currently, at Oakland Children's Hospital, our protocol utilizes the combined CSF cine, bSSFP, and dynamic bSSFP cine studies in patients with newly diagnosed hydrocephalus, those with shunt failure who are being considered for ETV, and those with idiopathic syringomyelia. Future prospective studies can be designed to elucidate the frequency of adhesion or lesion detection with these sequences in presumed communicating hydrocephalus and idiopathic syringomyelia.

Balanced SSFP and dynamic bSSFP cine are exciting imaging tools that allow clinicians and researchers to visualize "invisible" lesions of the central nervous system. These imaging techniques can be extremely useful in evaluating patients with disorders of CSF flow dynamics.

\section{References}

1. Bansmann PM, Sénégas J, Muellerleile K, Lund G, Kemper J, Adam G, et al: Assessment of left ventricular function parameters with a new three-dimensional shape model. Rofo 181:161-168, 2009

2. Barkhausen J, Ruehm SG, Goyen M, Buck T, Laub G, Debatin JF: MR evaluation of ventricular function: true fast imaging with steady-state precession versus fast low-angle shot cine MR imaging: feasibility study. Radiology 219:264-269, 2001

3. Cinalli G, Sainte-Rose C, Kollar EM, Zerah M, Brunelle F, Chumas P, et al: Hydrocephalus and craniosynostosis. J Neurosurg 88:209-214, 1998

4. Dilli A, Ayaz UY, Karabacak OR, Tatar IG, Hekimoglu B: Study of the left renal variations by means of magnetic resonance imaging. Surg Radiol Anat 34:267-270, 2012

5. Fischbach F, Thormann M, Seidensticker M, Kropf S, Pech M, Ricke J: Assessment of fast dynamic imaging and the use of Gd-EOB-DTPA for MR-guided liver interventions. J Magn Reson Imaging 34:874-879, 2011

6. Gottschalk A, Schmitz B, Mauer UM, Bornstedt A, Steinhoff S, Danz B, et al: Dynamic visualization of arachnoid adhesions in a patient with idiopathic syringomyelia using highresolution cine magnetic resonance imaging at 3T. J Magn Reson Imaging 32:218-222, 2010

7. Hirai T, Korogi Y, Shigematsu Y, Sugahara T, Takahashi M, Ushio Y, et al: Evaluation of syringomyelia with three-dimensional constructive interference in a steady state (CISS) sequence. J Magn Reson Imaging 11:120-126, 2000

8. Iskandar BJ, Hedlund GL, Grabb PA, Oakes WJ: The resolution of syringohydromyelia without hindbrain herniation after posterior fossa decompression. J Neurosurg 89:212-216, 1998

9. Jung NY, Moon WJ, Lee MH, Chung EC: Magnetic resonance cisternography: comparison between 3-dimensional driven equilibrium with sensitivity encoding and 3-dimensional balanced fast-field echo sequences with sensitivity encoding. J Comput Assist Tomogr 31:588-591, 2007

10. Klekamp J: Treatment of syringomyelia related to nontraumatic arachnoid pathologies of the spinal canal. Neurosurgery 72:376-389, 2013

11. Koizumi J, Horie T, Muro I, Kimura E, Shimizu K, Orii M, et al: Magnetic resonance venography of the lower limb. Int Angiol 26:171-182, 2007

12. Miller J, Acar F, Hamilton B, Burchiel K: Preoperative visualization of neurovascular anatomy in trigeminal neuralgia. $\mathbf{J}$ Neurosurg 108:477-482, 2008

13. Moon WJ, Roh HG, Chung EC: Detailed MR imaging anatomy of the cisternal segments of the glossopharyngeal, vagus, and spinal accessory nerves in the posterior fossa: the use of 3D balanced fast-field echo MR imaging. AJNR Am J Neuroradiol 30:1116-1120, 2009

14. Nakai T, Yamamoto H, Tanaka K, Koyama J, Fujita A, Taniguchi M, et al: Preoperative detection of the facial nerve by high-field magnetic resonance imaging in patients with vestibular schwannoma. Neuroradiology 55:615-620, 2013

15. Nasu K, Kuroki Y, Kuroki S, Murakami K, Nawano S, Moriyama N: Optimization of Gd-DTPA-enhanced balanced turbo field echo sequence in abdominal imaging: clinical application. Magn Reson Med Sci 3:73-77, 2004

16. Roser F, Ebner FH, Danz S, Riether F, Ritz R, Dietz K, et al: Three-dimensional constructive interference in steady-state magnetic resonance imaging in syringomyelia: advantages 
over conventional imaging. J Neurosurg Spine 8:429-435, 2008

17. Soo TM, Sandquist L, Tong D, Barrett R: Surgical treatment of idiopathic syringomyelia: silastic wedge syringosubarachnoid shunting technique. Surg Neurol Int 5:114, 2014

18. Sumi T, Sumi M, Van Cauteren M, Kimura Y, Nakamura T: Parallel imaging technique for the external carotid artery and its branches: comparison of balanced turbo field echo, phase contrast, and time-of-flight sequences. J Magn Reson Imaging 25:1028-1034, 2007

19. Tsuchiya K, Aoki C, Hachiya J: Evaluation of MR cisternography of the cerebellopontine angle using a balanced fast-field-echo sequence: preliminary findings. Eur Radiol 14:239-242, 2004

\section{Author Contributions}

Conception and design: Wang, Sun. Acquisition of data: Wang, Martin. Analysis and interpretation of data: Wang. Drafting the article: Wang. Critically revising the article: all authors. Reviewed submitted version of manuscript: all authors. Approved the final version of the manuscript on behalf of all authors: Wang. Study supervision: Sun.

\section{Supplemental Information Videos}

Video 1, Media Player. http://mfile.akamai.com/21490/wmv/ digitalwbc.download.akamai.com/21492/wm.digitalsource-naregional/peds13-620_video_1.asx.
Video 1, Quicktime. http://mfile.akamai.com/21488/mov/ digitalwbc.download.akamai.com/21492/qt.digitalsource-global/ peds13-620_video_1.mov.

Video 2, Media Player. http://mfile.akamai.com/21490/wmv/ digitalwbc.download.akamai.com/21492/wm.digitalsource-naregional/peds13-620_video_2.asx.

Video 2, Quicktime. http://mfile.akamai.com/21488/mov/ digitalwbc.download.akamai.com/21492/qt.digitalsource-global/ peds13-620_video_2.mov.

Video 3, Media Player. http://mfile.akamai.com/21490/wmv/ digitalwbc.download.akamai.com/21492/wm.digitalsource-naregional/peds13-620_video_3.asx.

Video 3, Quicktime. http://mfile.akamai.com/21488/mov/ digitalwbc.download.akamai.com/21492/qt.digitalsource-global/ peds13-620_video_3.mov.

Video 4, Media Player. http://mfile.akamai.com/21490/wmv/ digitalwbc.download.akamai.com/21492/wm.digitalsource-naregional/peds13-620_video_4.asx.

Video 4, Quicktime. http://mfile.akamai.com/21488/mov/ digitalwbc.download.akamai.com/21492/qt.digitalsource-global/ peds13-620_video_4.mov.

\section{Correspondence}

Doris D. Wang, Department of Neurological Surgery, University of California, San Francisco, 505 Parnassus Ave., Rm. 779M, San Francisco, CA 94143-0112. email: wangd@ neurosurg.ucsf. edu. 